\title{
Correlation between Body Mass Index, Thyroid Function Test and Neck Ultrasound in Euthyroid and Thyroid Disorder patients: a Centre Based Retrospective Study
}

\author{
Tirtha Lal Upadhyaya ${ }^{1}$, Shyam Sundar Parajuly ${ }^{2}$, Dilasma Gharti Magar ${ }^{3}$, Roshan Pangeni ${ }^{2}$ \\ ${ }^{1}$ Head of the Department, Department of Medicine, Gandaki Medical College and Teaching Hospital, \\ Pokhara, and Director of Diabetes, Thyroid and Endocrinology Care Centre, New Road Pokhara, Nepal, \\ ${ }^{2}$ Department of Radiology, Western Regional Hospital, Pokhara Academy of Health and Sciences, \\ Pokhara, Nepal, ${ }^{3}$ Department of Pathology, Manipal Teaching Hospital, Pokhara, Nepal
}

\begin{abstract}
Background: Thyroid dysfunction is a major health issue among the Nepalese population. It is the prone region for iodine deficiency disease. Altered living status, sub-urbanization and behavioral factors among the people are believed to be thyroid sufferers. The effects of hypothyroidism and hyperthyroidism on body weight have been clinically clearly demonstrated however there are only few literatures published showing the relationship between BMI and thyroid function among the two groups of population (euthyroid and thyroid dysfunction).

Objective: This study aims to study the relationship of TSH (thyroid stimulating hormone) and thyroid hormones with BMI and neck ultrasound findings in euthyroid and thyroid dysfunction subjects. Methods: This is a centre based retrospective study carried out in Diabetes, Thyroid and Endocrinology Care Centre (DTECC), Pokhara Nepal. The study used the archives of the laboratory and patient clinical information on the Centre during the years 2016 March to 2017 March. Detailed information regarding patientís age, medical history and previous history of smoking and alcohol consumption data, patients BMI (Body Mass Index) and neck ultrasound findings were obtained.

Results: Of the total five hundred and six cases, four hundred and forty cases were females and sixty six cases were male. 59.4 percentages of the cases had increased body mass index and about 48 percentages of the total cases had abnormal thyroid function tests. Of the total, neck ultrasound revealed findings suggestive of Hashimoto Thyroiditis in two hundred and forty four cases.

Conclusion: Thyroid dysfunction is associated with some components of metabolic syndrome. The thyroid function disorders in conjunction with the strong influence of various environmental factors can increase body weight and lead to obesity. Increased in BMI has strong influence on thyroid hormone level.
\end{abstract}

Key Words: Body Mass Index, Euthyroid, Thyroid dysfunction,

\section{Introduction:}

Thyroid dysfunctions are the second most common endocrine disease, being next only to diabetes mellitus. Depending upon the level of thyroid hormones in blood it manifests ranging from hyperthyroidism to hypothyroidism. ${ }^{1}$ Abnormal

Corresponding Address: Dr. Tirtha Lal Upadhyaya, MD Associate Professor, Head of the Department, Department of Medicine, Gandaki Medical College \& Teaching Hospital, Pokhara, Nepal, E-mail: tirtha77@hotmail.co
Thyroid functions can be induced either by excessive or deficient of iodine intake. Study shows that almost one-third of the world's population lives in areas of iodine deficiency. ${ }^{2}$ Dietary Iodine deficiency is an important underlying cause of thyroid dysfunction, especially among the people who resides in hilly region. ${ }^{3}$

Thyroid dysfunction is a major health issue among the Nepalese population as it is the country of 
Himalaya, landlocked and surrounded by two giant countries China and India; is the prone region for iodine deficiency disease. Altered living status, sub-urbanization and behavioral factors among the people are believed to be thyroid sufferers. Lack of country's census in regarding the prevalence of thyroid suffers and government policy for prevention and disease control, lots of information and the proper data to compare the study is beyond our dream. However, few research data has been published in different parts of the Nepal regarding the prevalence rate and status..$^{1,4,5}$

This study aims to correlate the relationship of TSH (thyroid stimulating hormone) and thyroid hormones with BMI and at the same time its correlation with the neck ultrasound findings.

\section{Methods:}

This is a centre based retrospective study carried out in Diabetes, Thyroid and Endocrinology Care Centre (DTECC), Pokhara Nepal. Patients who visited the centre during the period, 2016 March to 2017 March, either for thyroid screening purpose or for medication purpose was randomly included. Irrespective of patientís age, cast and ethnicity patients were recruited. Data were collected from the archives of the laboratory and patient clinical informationís file. Detailed information regarding patientís age, medical history and previous history of smoking and alcohol consumption data, patients BMI (Body Mass Index), fasting serum thyroid function test report and neck ultrasound findings were obtained. Sonographic findings of the neck ultrasound were further compared with the clinical diagnosis and serum thyroid report. Ethical permission for the study was obtained from the board of DTECC. Descriptive analysis was done for statistical interpretations. All the data were first input in Microsoft Excel software (version 2003) which was further analyzed by SPSS, Version 23 (Chicago, USA).

\section{Results}

There were in total, five hundred and six patients who fulfilled the criteria for the study were enrolled. The patient mean age was 42.43 years, ranging from 8 to 88 years. There were four hundred and forty female cases $(87 \%)$ and only sixty-six male cases (13\%). Female predominance was common (Figure 1). Thyroid dysfunction was more common in women above forty years of age.

\section{main-title}

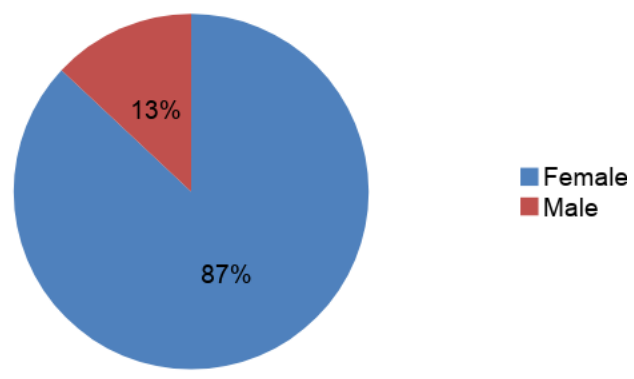

\section{Figure 1 Male and female participant for the study}

Most of the patients who visited the centre for thyroid dysfunction examination or for screening purposes revealed obese status. Of the five hundred and six cases, forty percentage of cases had normal Body Mass Index $(<25)$, thirty six percentage of the total cases were overweight (25-30). Similarly $19 \%$ of the cases were categorized as obese (30$35)$. Only $5 \%$ of the total cases $(24 / 506)$ were categorized under morbidity (Figure 2).

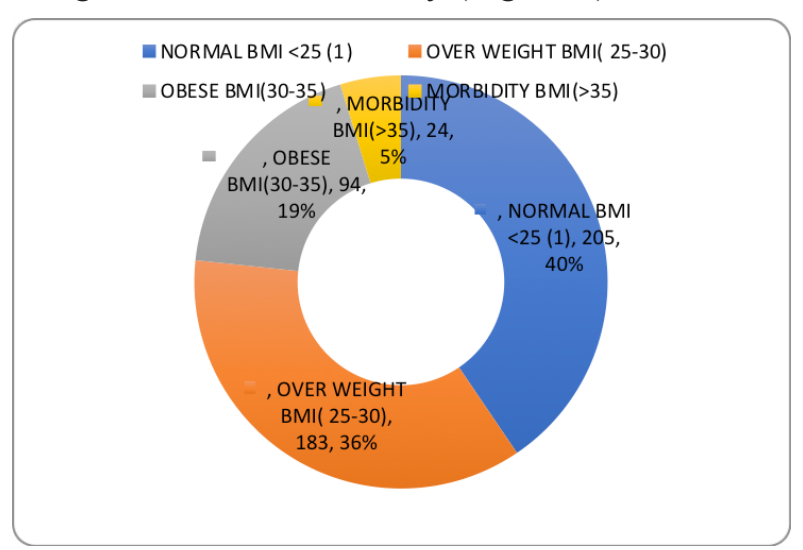

Figure 2 Levels of BMI 
Our study revealed that of the total 506 cases that had serum thyroid function test in our centre using CLIA(Chemiluminescence Immunoassay) methods in fasting status had high TSH in one hundred and ninety-seven cases ( $38.93 \%)$, low in 57 cases $(11.26 \%)$ and normal in 252 cases ( 49.80\%) (Figure 3).



Figure 3. TSH levels distribution in the population

Male and female distribution of serum thyroid hormone level as per the biochemistry report were further categorized as low, normal and high as shown in table 1.

Table 1. Distribution of Thyroid serum TSH levels in male and female population

\begin{tabular}{|c|c|c|c|c|}
\hline \multirow{2}{*}{} & \multicolumn{4}{|c|}{ SERUM TSH VALUES } \\
\cline { 2 - 5 } & Low & Normal & High & $\begin{array}{c}\text { Total } \\
(\mathrm{n}=506)\end{array}$ \\
\hline $\begin{array}{c}\text { Male } \\
(\mathrm{n}=66)\end{array}$ & $\begin{array}{c}6 \\
(9.0 \%)\end{array}$ & $\begin{array}{c}28 \\
(42.42 \%)\end{array}$ & $\begin{array}{c}32 \\
(48.48 \%)\end{array}$ & 66 \\
\hline $\begin{array}{l}\text { Female } \\
(\mathrm{n}=440)\end{array}$ & $\begin{array}{c}51 \\
(11.59 \%)\end{array}$ & $\begin{array}{c}224 \\
(50.90 \%)\end{array}$ & $\begin{array}{c}165 \\
(37.5 \%)\end{array}$ & 440 \\
\hline
\end{tabular}

All of the enrolled cases underwent neck ultrasound. Neck ultrasound was performed in the transverse and longitudinal planes using an Esaote Mylab 90 US scanner equipped with $7.5-13.0 \mathrm{MHz}$ linerarray transducer. Sonographic features of thyroid parenchyma, vascualarity, presence or absence of lesion, features of lesions ( size, shape, margin, calcification etc), presence/ absence of lymph node enlargement, presence/absence of intra-tumoral blood flow etcetra were recorded according to the guidelines provided by American College of Radiology (ACR) TIRADS (Thyroid Image Reporting and Data System) 2017. ${ }^{6}$ Ultrasound findings for all the cases were shown in below diagram (Figure 4). The most common finding on ultrasound was Hashimoto Thyroidtis. 48.22 percentages of the cases had features of Hashimoto Thyroidtis (HT) (Figure 5). Sonographic features of HT typically showed diffusely altered parenchyma, with heterogeneously hypoechoic and echogenic septations with oftenly mild enlargement of thyroid gland with increased in parenchmyal vascularity. ${ }^{7-10}$

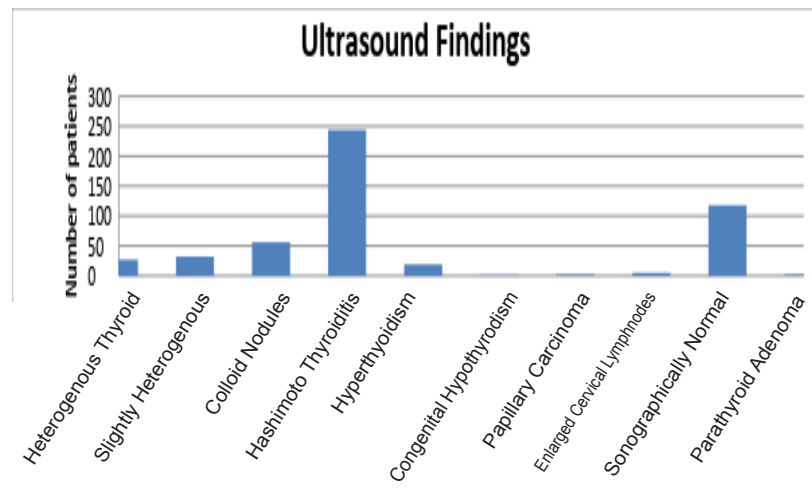

Figure 4. Illustrating the ultrasound findings

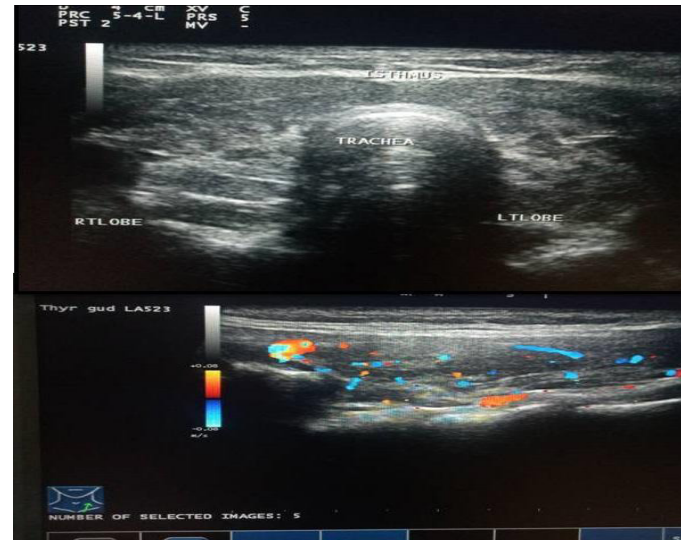

Figure 5. Neck ultrasound showing the typical appearance of Hashimoto Thyroiditis on ultrasound.

Transverse scan image A revealed diffusely altered parenchyma with heterogeneously hypoechoic and echogenic septations. Image B showing increased in parenchymal vascularity on Color Doppler imaging. 
Similarly, below figure showed the relation of BMI with thyroid function test. TSH increases as BMI increases. Most of the disorder or increased in TSH was noticed in obese group, however no normal thyroid function was noticed in morbidity group (BMI>35)

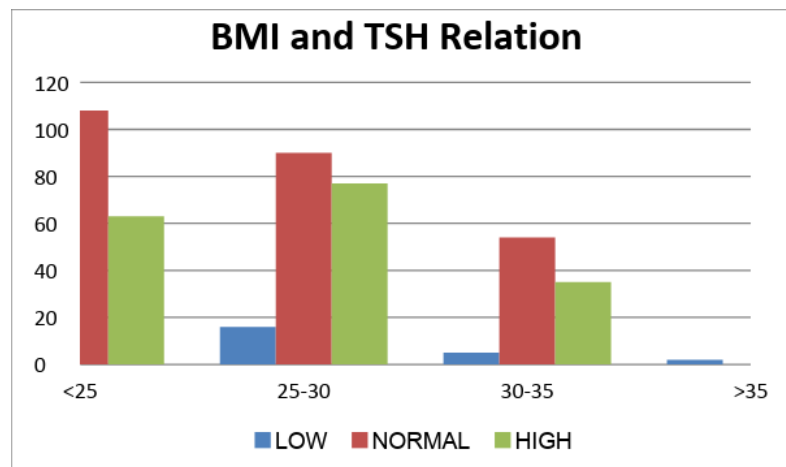

Figure 6. Relation of BMI and TSH

\section{Discussion}

Obesity and Overweight are defined as an excessive or abnormal fat accumulation in body that presents a risk to health. Generally, obesity of the body is measured by the body mass index (BMI), a person ís weight (in kilograms) divided by the square of his or her height (in meters). A person with a BMI of 30 or more is generally considered obese. A person with a BMI equal to or more than 25 is considered overweight. ${ }^{11}$ A BMI of $<18.5$ is considered underweight while BMI $>=30$ is considered obese. Obesity leads to the risk for different diseases, study revealed that etiology of obesity is an imbalance between the energy ingested in food and the energy expanded. ${ }^{12}$

Drastic changes in life style, living style, socioeconomic status and adopting western culture might be one of the cause for obesity Thyroid hormones regulates metabolic processes essential for normal growth and development as well as regulating metabolism in the adult. It is well established that thyroid hormone status correlates with body weight and energy expenditure. ${ }^{13}$ Our study revealed that there is a strong relationship between body mass index and TSH as given in figure 6. As BMI increases, the level in TSH hormone increases leading to hypothyroidism as similar to study conducted by Fathima et al in $2016 .{ }^{14}$ In our study as BMI is over 35 , no cases had normal TSH values. However, we have also found that normal thyroid function was found in 54 cases with obese which was quite similar to study by Strata et al,. ${ }^{15}$ There he found that thyroid function was normal in obese. There was no normal TSH value in morbidity group , >35 BMI; Approximately $92 \%$ of the cases had increased TSH levels which was quite opposite to the Strata et al 15 and similar findings to other study. ${ }^{16,17}$

Recent advances in ultrasound technology have enhanced anatomical characterization of neck pathology offering higher diagnostic accuracy leading to an eminent role in lesion characterization and clinical decision making. ${ }^{18}$ Ultrasound evaluation of thyroid gland is economically and technically a very convenient tool in assessing and evaluating the morphological features of thyroid nodules. Sometimes clinical and laboratory findings are not sufficient for identification of nature of lesions especially when they are non palpable. ${ }^{19}$ Emerging techniques and recent advances of piezoelectric crystal with high definition of ultrasound probe has brought the revolution for even few millimeter of thyroid microcalcifcation..$^{20}$ As illustrated in figure 4 and figure 5, Hashimoto thyroiditis is the most common cause for thyroid dysfunction leading to hypothyroidism which is very similar to study conducted by Adhikari BR et al. ${ }^{21}$ Female dominant with age more than 40 years old was seen in our population as well as in other study. ${ }^{22,23}$

\section{Conclusions}

Thyroid dysfunction is increasing throughout the world. Increased in BMI has strong influence on thyroid hormone level. Screening of thyroid function test is mandatory. Simple ultrasound tool can be very helpful diagnostically in determining the nature of lesion when clinically and laboratory findings creates dilemma.

\section{Limitations}

Our study was single institutional based centre. 
Correlation between BMI and TFT...

Jour of Diab and Endo Assoc of Nepal 2018; 2 (2):(3-7)

Sample size was good for this study; however more sample size would have been better to represent the entire western population to decrease the biasness. Other laboratory parameters like fT3, fT4, thyroglobulin, anti-thyroperoxidase, antithyroglobulin, TSH receptor antibodies would have been included to rule out thyroid disorders. As this is the preliminary study in western part of Nepal, we certainly will bring some good research paper regarding the thyroid dysfunction very soon.

\section{REFERENCES}

1. Dangol R, Lanjekar P, Pulipati C. Thyroid Function Abnormalities among Hospital Patients of Hilly Nepal. J Lumbini Med Coll . 2018; 5(1):29 -33.

2. Zimmerman MB. Iodine deficiency. Endocr Rev 2009; (30):376-408.

3. Effects of increased iodine intake on thyroid disorders. Sun X, Shan Z, Teng W. Endocrinol Metab.2014; 29(3):240-7.

4. Regmi A, Shah B, Rai BR, et al. Serum lipid profile in patients with thyroid disorders in central Nepal. Nepal Med Coll J. 2010;12:253ñ6.

5. Risal P, Maharjan BR, Koju R, et al. Variation of total serum cholesterol among the patient with thyroid dysfunction. Kathmandu Univ Med J. 2010;8:265ñ68.

6. Tessler FN, Middleton WD, Grant EG, Hoang JK, Berland LL, Teefey SA et al. ACR Thyroid Imaging, Reporting and Data System (TI-RADS): White paper of the ACR TI-RADS Committee. J Am Coll Radiol 2017; 14(5):587-95.

7. YehHC, Futterweit W, Gilbert P. Micronodulation: ultrasonographic sign of Hashimoto thyroiditis. J Ultrasound Med 1996; 15:813 9 .

8. Simeone JF, Daniels GH, Mueller PR, et al. Highresolution real-time sonography of the thyroid. Radiology 1982; 145:431 5.

9. Butch RJ, Simeone JF, Mueller PR. Thyroid and parathyroid ultrasonography. Radiol Clin North Am 1985; 23:57 71 .

10. Pedersen OM, Aardal NP, Larssen TB, Varhaug JE, Myking O, Vik-Mo $\mathrm{H}$. The value of ultrasonography in predicting autoimmune thyroid disease. Thyroid 2000; 10:2519.
11. http://www.who.int/topics/obesity/en/ cited online on August 112018.

12. Reinehr T, Andler W. Thyroid hormones before and after weight loss in obesity. Arch Dis Child 2002; 87(4):320-3.

13. Mullur R, Liu Y-Y, Brent GA. Thyroid Hormone Regulation of Metabolism. Physiological Reviews. 2014; 94(2):355-382.

14. Faazila Fathima, Preetha. Evaluation of Thyroid Function Test in Obese Patients. Asian J Pharm Clin Res. 2016;9(3):353-5.

15. Strata A, Ugolotti G, Contini C, Magnati G, Pugnoli C, Tirelli F, et al. Thyroid and obesity: survey of some function tests in a large obese population. Int J Obes 1978; 2(3):333-40.

16. Rimm AA, Werner LH, Yserloo BV, Bernstein RA. Relationship of obesity and disease in 73,532 weight-conscious women. Public Health Rep 1975; 90(1):44-54.

17. Solanki A, Bansal S, Jindal S, Saxena V, Shukla US. Relationship of serum thyroid stimulating hormone with body mass index in healthy adults. Indian Journal of Endocrinology and Metabolism. 2013;17(1):167-9.

18. Perros P, Boelaert K, Colley S, et al. Guidelines for the management of thyroid cancer. Clin Endocrinol (Oxf) 2014; 81(Suppl. 1):1-122.

19. Ajay K Goutam, Avadhesh P S Kushwah, Sonjjay Pande. Ultasonography and CT evaluation of neck masses. International Journal of Contemporary Medical Research 2017;4(6):1392-97.

20. Zhou Q, Lam KH, Zheng H, Qiu W, Shung KK. Piezoelectric single crystals for ultrasonic transducers in biomedical applications. Progress in materials science. 2014;66:87-111.

21. Adhikari BR, Twayana RS, Shrestha S, Vaidya N, Aghrahari M , Ghimire B. Pattern of thyroid disorders in people from central Nepal: A Hospital based study. International Journal of Scientific and Research Publications, 2017; 7(8):132-5.

22. Wang C, Crapo LM. The epidemiology of thyroid disease and implications for screening. Endocrinol Metab Clin North Am. 1997; 26(1):189-218.

23. Vanderpump MP and Tunbridge WM. Epidemiology and prevention of clinical and subclinical hypothyroidism. Thyroid. 2002; 12: 839-47. 